\title{
Additive nonparametric regression estimation via backfitting and marginal integration: Small sample performance*
}

\author{
Fernando A. Boeira Sabino da Silva**
}

\begin{abstract}
In this paper, we conducted a Monte Carlo investigation to reveal some characteristics of finite sample distributions of the Backfitting (B) and Marginal Integration (MI) estimators for an additive bivariate regression. We are particularly interested in providing some evidence on how the different methods for the selection of bandwidth, such as the plug-in method, influence the finite sample properties of the MI and B estimators. We are also interested in providing evidence on the behavior of different bandwidth estimators relatively to the optimal sequence that minimizes a chosen loss function. The impact of ignoring the dependency between regressors is also investigated. Finally, differently from what occurs at the present time, when the B and MI estimators are used ad-hoc, our objective is to provide information that allows for a more accurate comparison of these two competing alternatives in a finite sample setting.
\end{abstract}

Key Words: Additive nonparametric regression, Local polynomial estimation, Automatic bandwidth selection, Backfitting estimation, Marginal integration.

JEL Code: C14, C15.

* Thanks to Cristiano Fernandes, Marcelo Côrtes Neri and an anonymous referee for their comments and suggestions and Carlos Martins-Filho for the useful contributions throughout the article. The author holds responsibility for any remaining errors.

** Department of Statistics, Federal University of Rio Grande do Sul, Av. Bento Gonçalves, 9500, Porto Alegre, RS, 91509-900, Brazil, e-mail: fsabino@mat.ufrgs.br and fsabino@fgumail.br.

$\overline{\text { Brazilian Review of Econometrics Rio de Janeiro v.22, } \mathrm{n} \text { 을,pp.275-298 Nov.2002 }}$ 
Additive nonparametric regression estimation

\section{Resumo}

Neste artigo realizou-se um experimento de Monte Carlo para revelar algumas características das distribuiçōes em amostras finitas dos estimadores Backfitting (B) e de Integração Marginal (MI) para uma regressão aditiva bivariada. Está-se particularmente interessado em fornecer alguma evidência de como os diferentes métodos de seleção da janela $h_{n}$, tais como os métodos plug-in, impactam as propriedades em pequenas amostras dos estimadores. Está-se interessado, também, em fornecer evidência do comportamento de diferentes estimadores de $h_{n}$ relativamente a seqüência ó tima de $h_{n}$ que minimiza uma função perda escolhida. $O$ impacto de ignorar a dependência entre os regressores na estimação da janela é também investigado. Esta é uma prática comum e deve ter impacto sobre o desempenho dos estimadores. Por fim, diferentemente do que ocorre atualmente, quando a utilização dos estimadores-B e MI é feita de maneira completamente ad-hoc, há o objetivo de fornecer a usuários informação que permita uma escolha mais objetiva de qual estimador usar quando se está trabalhando com uma amostra finita.

\section{Introduction.}

The estimation of additive nonparametric regressions has been recently discussed in several studies. The hypothesis of additivity is of practical and theoretical interest. From a practical viewpoint, this supposition facilitates interpretation and reduces the computational demand for an unrestricted nonparametric regression. Theoretically speaking, it guarantees rates of convergence for nonparametric estimators that are reasonably quick and independent from the dimensionality problem identified by Friedman \& Stuetzle(1981). In addition, with this hypothesis, there is no need to assume some kind of hardly justifiable metric when the variables are measured in different units or are highly correlated (Buja, Hastie \& Tibshirani, 1989). Currently, there are two viable estimators for an additive nonparametric model - the Backfitting estimator (B-estimator) and the Marginal Integration estimator (MI-estimator). The B-estimator is based on Friedman \& Stuetzle(1981); however, it became popular through the studies carried out by Hastie \& Tibshirani $(1986,1990)$. Its proper- 
ties were studied in Buja, Hastie \& Tibshirani(1989) and Opsomer \& Ruppert(1997). At present, little is known about the statistical properties of the B-estimator. In general, it is still not possible to construct asymptotically valid confidence intervals for the estimated regression, even when the bandwidth $h_{n} \rightarrow 0$ at a desired rate. The knowledge about the B-estimator properties is even scarcer, when $h_{n}$ is chosen by minimizing the criterion functions most widely used in the literature. Consequently, in practice, little is known about the asymptotic properties and in finite samples of the B-estimator. The MI-estimator was introduced in the seminal articles written by Linton \& Nielsen(1995) and Linton \& Härdle(1996). One of the most attractive properties of the MI-estimator is that it can be shown to be asymptotically normal when the regressor specific bandwidth $h_{n}$ converges to zero at a preset rate. Nevertheless, its asymptotic distribution is still unknown when $h_{n}$ is chosen by data driven methods currently available in the literature, such as cross validation and several plug-in methods, including those proposed by Silverman(1986) and Opsomer \& Ruppert(1998). The difficulty in establishing the asymptotic normality in this setting is two-fold. Firstly, data driven $h_{n}$ are stochastic sequences that may interact detrimentally with regressors and the regressand, which creates an additional difficulty in establishing the asymptotic normality of the MII-estimator. Secondly, data driven $h_{n}$ are chosen by minimizing a criterion function (loss or risk). For the most widely used criterion functions, the resulting optimal sequence of $h_{n}$, do not converge to zero at the rate that is necessary to obtain asymptotic normality. Just like the B-estimator, little is known, in practice, about both asymptotic and finite sample distributional properties of the MI-estimator.

To make currently available (asymptotic) distributional results useful we have to adapt them to the case in which $h_{n}$ is a data dependent stochastic sequence. An alternative is to provide experimental 
evidence of the performance of the estimators based on several methods for the selection of bandwidth $h_{n}$ by means of a Monte Carlo investigation. Therefore, in this paper, we will conduct a Monte Carlo investigation in order to show some characteristics of the distributions in finite samples of B and MI-estimators for an additive bivariate regression. We are particularly interested in providing some evidence of how the different methods for the selection of bandwidth $h_{n}$, such as plug-in methods, impact the finite sample properties of these estimators. Also, we attempt to offer some evidence of the behavior of different estimators of $h_{n}$ relatively to the optimal sequence of $h_{n}$ that minimizes a chosen loss function. The impact of ignoring the dependency between regressors in the estimation of the bandwidth is also investigated. This is common practice and should impact estimators' performance. Finally, differently from what occurs currently when the B and MI-estimators are used ad-hoc, the aim is to provide users with information that allows for a more accurate selection of which estimator should be used in a finite sample setting. Besides this introduction the paper has five more sections. Section 2 describes the specification of the model and the two estimators under analysis in a unified format. Section 3 describes the methods for the selection of bandwidth $h_{n}$ under study. Section 4 presents the data-generating process to be used in the Monte Carlo investigation. Section 5 discusses the results of the analysis. Section 6 provides a brief conclusion.

\section{Specification of the Model and the Estimators under Analysis.}

The statistical model considered herein is that of a bivariate additive nonparametric regression adjusted by a local linear smoother. It is assumed that $\left\{\left(y_{t}, x_{t}, z_{t}\right)^{\prime}\right\}_{t=1}^{n}$ form a sequence of realizations of a random vector $I I D(Y, X, Z)$ with $E\left(Y \mid X=x_{t}, Z=z_{t}\right)=$ 
$m_{1}\left(x_{t}\right)+m_{2}\left(z_{t}\right)$ and $V\left(Y \mid X=x_{t}, Z=z_{t}\right)=\sigma^{2} . m_{1}(\cdot)$ and $m_{2}(\cdot)$ are real valued functions with some regularity conditions (see Buja, Hastie \& Tibshirani, 1989), including a suitably chosen degree of differentiability. It is convenient for our purposes to define the following vectors: $Y=\left(Y_{1}, \ldots, Y_{n}\right)^{\prime}, X=\left(X_{1}, \ldots, X_{n}\right)^{\prime}$, $Z=\left(Z_{1}, \ldots, Z_{n}\right)^{\prime}, \vec{m}_{1}(X)=\left(m_{1}\left(X_{1}\right), \ldots, m_{1}\left(X_{n}\right)\right)^{\prime}, \vec{m}_{2}(Z)=$ $\left(m_{2}\left(Z_{1}\right), \ldots, m_{2}\left(Z_{n}\right)\right)^{\prime}, e_{t}^{k}=(0, \ldots, 1, \ldots, 0)^{\prime}$ is a vector of length $k$, where number one appears in the $t^{\text {th }}$ position of the vector, and for any constant $c, \vec{c}_{n}=(c, \ldots, c)^{\prime}$ is a vector of length $n$. We denote by $K_{d}: \Re^{d} \rightarrow \Re$ a $d$-variate symmetric kernel function with $d=1,2$ and by $h_{1 n}$ and $h_{2 n}$ the bandwidths associated with the estimation of $m_{1}$ and $m_{2}$, respectively. By using the previously introduced notation, define two estimating weight functions as:

$$
\mathbf{s}_{1}(x): \Re \rightarrow \Re^{n^{n}}: \mathbf{s}_{1}(x)=\mathbf{e}_{1}^{2^{\prime}}\left(R_{X}(x)^{\prime} V_{X}(x) R_{X}(x)\right)^{-1} R_{X}(x)^{\prime} V_{X}(x)
$$

and

$$
\mathrm{s}_{2}(z): \Re \rightarrow \Re^{\mathrm{n}}: \mathrm{s}_{2}(z)=\mathbf{e}_{1}^{2^{\prime}}\left(\mathbf{R}_{\mathrm{Z}}(z)^{\prime} \mathrm{V}_{\mathrm{Z}}(\mathrm{z}) \mathbf{R}_{\mathrm{Z}}(\mathrm{z})\right)^{-1} \mathbf{R}_{\mathrm{Z}}(\mathrm{z})^{\prime} \mathrm{V}_{\mathrm{Z}}(\mathbf{z})
$$

Let $\mathbf{S}_{1}$ and $\mathbf{S}_{2}$ represent the matrices whose rows are the smoothers at $\mathbf{X}$ and $\mathbf{Z}$ :

$$
\mathrm{S}_{1}=\left(\begin{array}{c}
\mathrm{s}_{1}\left(\mathrm{X}_{1}\right) \\
\cdot \\
\cdot \\
\cdot \\
\mathrm{s}_{1}\left(\mathrm{X}_{\mathrm{n}}\right)
\end{array}\right) \text { and } \mathrm{S}_{2}=\left(\begin{array}{c}
\mathrm{s}_{2}\left(\mathrm{Z}_{1}\right) \\
\cdot \\
\cdot \\
\cdot \\
\mathrm{s}_{2}\left(\mathrm{Z}_{\mathrm{n}}\right)
\end{array}\right)
$$

Define the vector of the values estimated at points $X_{1}, \ldots, X_{n}$ by $\widehat{\mathbf{m}}=\widehat{\mathbf{m}}_{1}+\widehat{\mathbf{m}}_{2}$, where $\widehat{\mathbf{m}}_{1}$ and $\widehat{\mathrm{m}}_{2}$ are the solutions to the following system of estimating equations: 


$$
\operatorname{det}\left[\begin{array}{ll}
\mathrm{I}_{\mathbf{n}} & \mathrm{S}_{1}^{*} \\
\mathrm{~S}_{2}^{*} & \mathbf{I}_{\mathbf{n}}
\end{array}\right] \operatorname{det}\left[\begin{array}{c}
\widehat{\mathbf{m}}_{1} \\
\widehat{\mathbf{m}}_{2}
\end{array}\right]=\operatorname{det}\left[\begin{array}{l}
\mathrm{S}_{1}^{*} \\
\mathrm{~S}_{2}^{*}
\end{array}\right] \mathrm{Y}
$$

where $\mathbf{I}_{\mathbf{n}}$ is an identity matrix of dimension $n$ and $\mathbf{S}_{\mathbf{d}}^{*}=$ $\left(\mathbf{I}_{\mathbf{n}}-\mathbf{1} \mathbf{1}^{\prime} / \mathbf{n}\right) \mathbf{S}_{\mathbf{d}}, d=1,2^{1}$. In practice, the system is solved by using the backfitting algorithm, however, in the bivariate case, when the local linear estimator is used, the backfitting algorithm converges to an explicit solution to $\overrightarrow{\mathrm{m}}_{1}(\mathrm{X})$ and $\overrightarrow{\mathrm{m}}_{2}(\mathbf{Z})$ given by

$$
\overrightarrow{\mathrm{m}}_{1}^{b}(\mathrm{X})=\left(\mathrm{I}_{\mathbf{n}}-\left(\mathrm{I}_{\mathbf{n}}-\mathrm{S}_{1}^{*} \mathrm{~S}_{2}^{*}\right)^{-1}\left(\mathrm{I}_{\mathbf{n}}-\mathrm{S}_{1}^{*}\right)\right) \mathrm{Y}
$$

and

$$
\overrightarrow{\mathrm{m}}_{2}^{b}(\mathrm{Z})=\left(\mathrm{I}_{\mathbf{n}}-\left(\mathrm{I}_{\mathbf{n}}-\mathrm{S}_{2}^{*} \mathrm{~S}_{1}^{*}\right)^{-1}\left(\mathrm{I}_{\mathbf{n}}-\mathrm{S}_{2}^{*}\right)\right) \mathrm{Y}
$$

if the inverses exist. The existence of these estimators and their stochastic properties are still, in general, unknown; however, by using the local linear estimator, Opsomer \& Ruppert(1997,1998) derived a series of results (for large samples), which is shown below. In our case, there is a solution if:

A1: The kernel $K$ is bounded, continuous, has compact support and its first derivate has a finite number of sign changes over its support. In addition, $\boldsymbol{\mu}_{j}(K) \equiv \int u^{j} K(u) d u=0$ for all odd $j$ and $\mu_{2}(K) \neq 0$.

A2: The densities $f(x, z), f_{X}(x)$ and $f_{Z}(z)$ are bounded, continuous and have compact support, and their first derivates have a finite number of sign changes over their supports. Also, $f_{X}(x)>0$ and $f_{Z}(z)>0$ for all $(x, z) \in \operatorname{supp}(f)$ and

${ }^{1}$ The adjustment of the smoothers is necessary to guarantee the uniqueness of the solutions (if they exist), see Hastie \& Tibshirani(1990). 
Fernando A. Boeira Sabino da Silva

$$
\sup \left|\frac{f(x, z)}{f_{X}(x) f_{Z}(z)}-1\right|<1
$$

A3: When $n \rightarrow \infty, h_{1 n}, h_{2 n} \rightarrow 0$ and $n h_{1 n} / \log (n)$, $n h_{2 n} / \log (n) \rightarrow \infty$.

A4: The second derivates of $m_{1}$ and $m_{2}$ exist and are bounded and continuous.

To define the MII estimator, consider $\widehat{\mathrm{m}}\left(x, z ; h_{1 n}, h_{2 n}\right)$ a real valued function defined by $\widehat{m}\left(x, z ; h_{1 n}, h_{2 n}\right)=e_{1}^{3^{\prime}}\left(\mathrm{X}(\mathrm{x}, \mathbf{z})^{\prime} \mathbf{W}(\mathbf{x}, \mathbf{z}) \mathrm{X}\right.$ $(x, z))^{-1} \mathrm{~W}(\mathrm{x}, \mathrm{z}) \mathrm{Y}$, where $\mathrm{X}(\mathrm{x}, \mathrm{z})=\left(\overrightarrow{\mathrm{1}}_{\mathrm{n}}, \mathrm{X}-\vec{x}, \mathbf{Z}-\vec{z}\right)$ and

$$
\mathrm{W}(x)=\operatorname{diag}\left\{\frac{1}{h_{1 n} h_{2 n}} K_{2}\left(\frac{1}{h_{1 n}}\left(X_{t}-x\right), \frac{1}{h_{2 n}}\left(Z_{t}-z\right)\right)\right\}_{t=1}^{n}
$$

Define the matrix

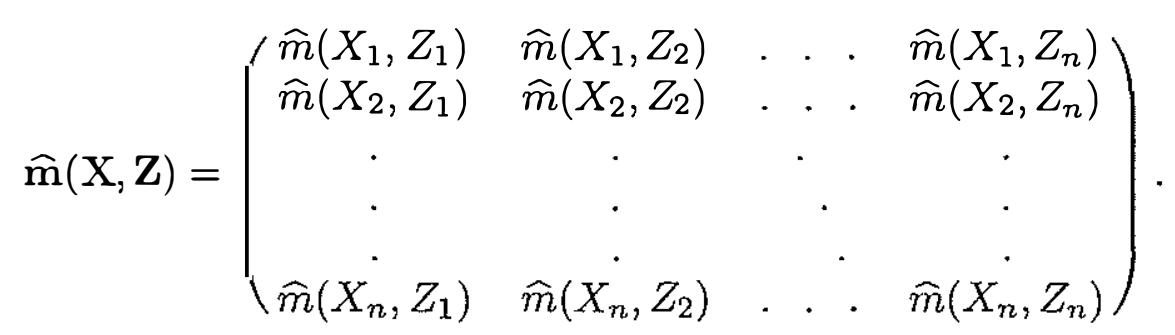

The MI-estimator for $\overrightarrow{\mathbf{m}}_{1}^{m i}(\mathrm{X})$ and $\overrightarrow{\mathbf{m}}_{2}^{m i}(\mathbf{Z})$, using the identity function as linking function and without considering an intercept (see Linton \& Nielsen,1995 and Linton \& Hardle, 1996), is respectively given by $\overrightarrow{\mathrm{m}}_{1}^{m i}(\mathrm{X})=\frac{1}{\mathrm{n}} \widehat{\mathrm{m}}(\mathrm{X}, \mathrm{Z}) \overrightarrow{1}_{\mathrm{n}}$, and $\overrightarrow{\mathrm{m}}_{2}^{m i}(\mathrm{Z})=\frac{1}{\mathrm{n}} \widehat{\mathrm{m}}(\mathrm{X}, \mathrm{Z})^{\prime} \overrightarrow{\mathrm{1}}_{\mathrm{n}}$. 
The weighting functions $Q_{1}$ and $Q_{2}$ (see Linton \& Nielsen, 1995) used for the estimation were the empirical distribution functions $F_{x n}(x)$ and $F_{z n}(z)$ that converge in distribution to $F_{X}(x)$ and $F_{Z}(z)$ respectively. The approximations provided in Linton \& Nielsen(1995, p.95) are still valid when the empirical functions are written in lieu of $Q$. Particularly, when $x$ and $z$ are independent, the empirical functions will be the optimal weighting functions in the sense that they minimize the variances of the asymptotic approximations.

The definitions provided above consider $h_{1 n}$ and $h_{2 n}$ as known nonstochastic sequences that converge to zero at a specified rate. For the B-estimator, Opsomer \& Ruppert(1997) show that when, $n \rightarrow \infty, h_{1 n}, h_{2 n} \rightarrow 0$ and $\frac{n h_{3 n}}{\log n}, \frac{n h_{2 n}}{\log n} \rightarrow \infty$ it is possible to obtain an asymptotic approximation to the conditional bias and conditional variance of $\vec{m}_{1}^{b}\left(X_{i}\right)$ and $\vec{m}_{2}^{b}\left(Z_{i}\right)$, where $\vec{m}_{1}^{b}\left(X_{i}\right)$ and $\vec{m}_{2}^{b}\left(Z_{i}\right)$ are the ith elements of $\overrightarrow{\mathbf{m}}_{1}^{b}(\mathrm{X})$ and $\overrightarrow{\mathbf{m}}_{2}^{b}(\mathbf{Z})$, respectively ${ }^{2}$. For the MIestimator, Linton \& Nielsen (1995) show that when $h_{1 n}, h_{2 n} \rightarrow 0$ and $n h_{1 n} h_{2 n}^{2}, n h_{2 n} h_{1 n}^{2} \rightarrow \infty$, then $\sqrt{n h_{1 n}}\left(\vec{m}_{1}^{m i}\left(X_{i}\right)-E\left(\vec{m}_{1}^{m i}\left(X_{i}\right)\right)\right)$ and $\sqrt{n h_{2 n}}\left(\vec{m}_{2}^{m i}\left(Z_{i}\right)-E\left(\vec{m}_{2}^{m i}\left(Z_{i}\right)\right)\right)$ are asymptotically normal, where $\vec{m}_{1}^{m i}\left(X_{i}\right)$ and $\vec{m}_{2}^{m i}\left(Z_{i}\right)$ are the ith elements of $\overrightarrow{\mathrm{m}}_{1}^{m i}(\mathrm{X})$ and $\overrightarrow{\mathrm{m}}_{2}^{m i}(\mathrm{Z})$, respectively ${ }^{3}$.

${ }^{2}$ The approximation is valid under another three suppositions (see Opsomer \& Ruppert,1997).

${ }^{3}$ It is possible to show that the data-driven bandwidth selection methods currently used in the literature, including cross validation and several plug-in methods, do not produce sequences $\left\{h_{1 n}\right\}$ and $\left\{h_{2 n}\right\}$ that converge to zero at the desired rates. For proofs, see Mlartins-Filho (2001). 


\section{Methods for Data Driven Bandwidth Selection.}

One of the most important steps in estimating the nonparametric regression models is the selection of smoothing parameters or bandwidths $h_{n}$. In essence, once the smoother is selected, the selection of the smoothing parameters is tantamount to the selection of the smooth itself (see Martins-Filho \& Bin, 1999 and Silva, 2001). In this paper, two methods for the automatic selection of the bandwidth $h_{n}{ }^{4}$ are considered. These two methods are variants of $p l u g$ in methods ${ }^{5}$, that use analytical optimization.

An appropriate error criterion (see Ruppert \& Wand, 1994 and Ruppert, Sheather \& Wand, 1995) is the weighted conditional MISE given by (in the case of $\mathrm{X}$ )

$$
\begin{aligned}
\operatorname{MISE}\left(\widehat{m}_{p}\left(\cdot ; h_{n}\right) \mid X_{1}, \ldots, X_{n}\right)= & E \int\left[\left\{\widehat{m}_{p}\left(x ; h_{n}\right)\right.\right. \\
& \left.-m(x)\}^{2} \mid X_{1}, . ., X_{n}\right] f_{X}(x) d x .
\end{aligned}
$$

where $f_{X}(x)$ represents the density of $X$ with support $[a, b]$. Also, assume that the errors are homoskedastic with variance $\sigma^{2}$. For $p$ odd Ruppert \& Wand(1994) show that

\footnotetext{
${ }^{4}$ The focus is on $h_{n}$ fixed within the support used.

${ }^{5}$ An alternative would be the use of cross validation. However, Jones, Marron \& Sheather(1996) comment that plug-in methods are better than cross validation methods, in simulation studies and asymptotically. The plug-in methods demand less computational time, do not show undersmoothing of the cross validation method, and the rate of convergence $\left(\widehat{h}_{n}-h_{n}\right) \rightarrow 0$ when $\widehat{h}_{n}$ is chosen by plug-in methods is quicker than the rate of convergence of $\left(\widetilde{h}_{n}-h_{n}\right) \rightarrow 0$ when $\widetilde{h}_{n}$ is obtained by cross validation.
} 


$$
\begin{aligned}
\operatorname{MISE}\left(\widehat{m}_{p}\left(\cdot ; h_{n}\right) \mid X_{1}, \ldots, X_{n}\right)= & {\left[\frac{h_{n}^{p+1} \mu_{p+1}(K(p))}{(p+1) !}\right]^{2} } \\
& \int m^{(p+1)}(x)^{2} f_{X}(x) d x+ \\
& \frac{R\left(K_{(p)}\right) \sigma^{2}(b-a)}{n h_{n}}+ \\
& o_{p}\left[h_{n}^{2 p+2}+\left(n h_{n}\right)^{-1}\right] .
\end{aligned}
$$

where $\mu_{j}(K)=\int u^{j} K(u) d u, K_{(p)}(u)=\left\{\left|M_{p}(u)\right| /\left|N_{p}\right|\right\} K(u), N_{p}$ is a matrix $(p+1) \times(p+1)$ whose $(i, j)$ th is equal to $\mu_{i+j-2}(K), M_{p}(u)$ is the same as $N_{p}$ but with the first column replaced by $\left(1, u, u^{2}, \ldots, u^{p}\right)^{\prime}$ and $R\left(K_{(p)}\right) \equiv \mu_{0}\left(K_{(p)}^{2}\right)$. The minimizer of $(6)$ is asymptotically

$$
\tilde{h}_{n}=\left[\frac{(p+1)(p !)^{2} R\left(K_{(p)}\right) \sigma^{2}(b-a)}{2 n \mu_{p+1}\left(K_{(p)}\right)^{2} \int m(p+1)(u)^{2} f_{X}(u) d u}\right]^{1 \mid(2 p+3)}
$$

if $\int m^{(p+1)}(u)^{2} f_{X}(u) d u$ is different from zero. A convenient error criterion, which uses only the fitted values at the observation points, is the conditional MASE, discussed by Hardle, Hall \& Marron(1988). In the univariate case, the MASE of $m$ can be written $a^{6}$

$$
\operatorname{MASE}\left(\widehat{m}_{p}\left(\cdot ; h_{n}\right) \mid X_{1}, \ldots, X_{n}\right)=\frac{1}{n} \sum_{i=1}^{n} E\left\{\left(\left\{\widehat{m}_{p}\left(x ; h_{n}\right)-m(x)\right\}^{2} \mid X_{1}, \ldots, X_{n}\right)\right\}
$$

\footnotetext{
${ }^{6}$ Note that (8) is a discrete approximation to (5).
} 
The basic principle of plug-in methods is the direct estimation of the estimates of $\sigma^{2}$ and of the functionals that appear in the expressions describing the values of the smoothing parameters $h_{n}$, after the criterion to be used for the nonparametric estimation has been minimized.

The plug-in method proposed in Linton \& Nielsen(1995) is based upon the following rule of thumb (ROT):

$$
h_{\text {inROT }}=\left\{\frac{\widetilde{\sigma}^{2} R\left(K_{(1)}\right)\left(b_{i}-a_{i}\right)}{\mu_{2}\left(K_{(1)}\right)^{2}\left(\widehat{\theta}_{1}+\widehat{\theta}_{2}\right)^{2}}\right\}^{1 / 5} n^{-1 / 5},
$$

where $i=1,2, b_{i}$ and $a_{i}$ denote the sample maximum and minimum of the regressor of interest, $\widehat{\theta}_{1}$ and $\widehat{\theta}_{2}$ are the coefficients of $x^{2} / 2$ and $z^{2} / 2$ obtained from an ordinary least-squares regression of $y$ on a constant, $x, z, x^{2} / 2, z^{2} / 2$ and $x z$, and $\tilde{\sigma}^{2}$ is obtained from the residuals of this regression. This rule is asymptotically optimal in terms of the AMISE criterion (see equation 7), when $p=1, x$ and $z$ are independent and the bivariate regression model $m(x, z)$ is a quadratic function. $\widehat{\theta}_{1}$ and $\widehat{\theta}_{2}$ are merely approximations to the second derivate that will appear in (7) when $p=1$.

Another plug-in method used was proposed in Opsomer \& Ruppert (1998). The aim, in this case, is to choose $h_{1 n}, h_{2 n} \in \Re$ such that

$\operatorname{MASE}\left(h_{1 n}, h_{2 n} \mid \mathbf{X}, \mathbf{Z}\right)=\frac{1}{\mathrm{n}} \sum_{\mathbf{i}=1}^{\mathbf{n}} \mathbf{E}\left(\widehat{\mathbf{m}}\left(\mathbf{X}_{\mathbf{i}}, \mathbf{Z}_{\mathbf{i}}\right)-\mathrm{m}\left(\mathbf{X}_{\mathbf{i}}, \mathbf{Z}_{\mathbf{i}}\right) \mid \mathbf{X}, \mathbf{Z}\right)^{2}$

Brazilian Review of Econometrics

22 (2) November 2002 
From the corollary 4.2 by Opsomer \& Ruppert (1997), the asymptotic approximation to the conditional MASE given in (10) above, when the additive model is fitted by local linear regression, denoted by $A M A S E$, is given by:

$$
\begin{aligned}
\operatorname{AMASE}\left(h_{1 n}, h_{2 n} \mid \mathbf{X}, \mathbf{Z}\right) & =\frac{\mu_{2}\left(K_{(1)}\right)^{2}}{4} \\
& \left(h_{1 n}^{4} \theta_{11}+h_{1 n}^{2} h_{2 n}^{2} \theta_{12}+h_{2 n}^{4} \theta_{22}\right)+ \\
& \sigma^{2} R\left(K_{(1)}\right)\left(\frac{b_{x}-a_{x}}{n h_{1 n}}+\frac{b_{z}-a_{z}}{n h_{2 n}}\right)
\end{aligned}
$$

where

$$
\begin{gathered}
\theta_{11}=\frac{1}{n} \sum_{i=1}^{n}\left(\mathbf{t}_{\mathbf{i}}^{\prime} \mathbf{D}^{2} \mathbf{m}_{\mathbf{1}}+\mathbf{v}_{\mathbf{i}}^{\prime} \mathbf{E}\left(\mathbf{m}_{\mathbf{1}}^{(2)}\left(\mathbf{X}_{\mathbf{i}}\right) \mid \mathbf{Z}\right)\right)^{2} \\
\theta_{22}=\frac{1}{n} \sum_{i=1}^{n}\left(\mathbf{v}_{\mathbf{i}}^{\prime} \mathbf{D}^{2} \mathbf{m}_{\mathbf{2}}+\mathbf{t}_{\mathbf{i}}^{\prime} \mathbf{E}\left(\mathbf{m}_{\mathbf{2}}^{(2)}\left(\mathbf{Z}_{\mathbf{i}}\right) \mid \mathbf{X}\right)\right)^{2} \\
\theta_{12}=\frac{1}{n} \sum_{i=1}^{n}\left(\mathbf{t}_{\mathbf{i}}^{\prime} \mathbf{D}^{2} \mathbf{m}_{1}+\mathbf{v}_{\mathbf{i}}^{\prime} \mathbf{E}\left(\mathbf{m}_{\mathbf{1}}^{(2)}\left(\mathbf{X}_{\mathbf{i}}\right) \mid \mathbf{Z}\right)\right) \\
\left(\mathbf{v}_{\mathbf{i}}^{\prime} \mathbf{D}^{2} \mathbf{m}_{\mathbf{2}}+\mathbf{t}_{\mathbf{i}}^{\prime} \mathbf{E}\left(\mathbf{m}_{\mathbf{2}}^{(2)}\left(\mathbf{Z}_{\mathbf{i}}\right) \mid \mathbf{X}\right)\right)^{2}
\end{gathered}
$$

and $t_{\mathbf{i}}^{\prime}$ and $\mathbf{v}_{\mathbf{j}}$ represent the ith row and the jth column of $\left(\mathbf{I}-\mathbf{T}_{12}^{*}\right)^{-1}$, provided the inverse matrix exists and $\left[\mathrm{T}_{12}^{*}\right]_{i j}=$ $\frac{1}{n} \frac{f_{X Z}\left(X_{i}, Z_{j}\right)}{f_{X}\left(X_{i}\right) f_{Z}\left(Z_{j}\right)}-\frac{1}{n}$.

By denoting the values of the bandwindths that minimize $A M A S E$ by $h_{1 n A M A S E}$ and $h_{2 n A M A S E}$ and under the assumption of independence between $\mathbf{X}$ and $\mathbf{Z}$, it is possible to write 


$$
h_{1 n A M A S E}=\left(\frac{R\left(K_{(1)}\right) \sigma^{2}\left(b_{1}-a_{1}\right)}{n \mu_{2}\left(K_{(1)}\right)^{2} \theta_{11}}\right)^{1 / 5}
$$

and

$$
h_{2 n A M A S E}=\left(\frac{R\left(K_{(1)}\right) \sigma^{2}\left(b_{2}-a_{2}\right)}{n \mu_{2}\left(K_{(1)}\right)^{2} \theta_{22}}\right)^{1 / 5} .
$$

The estimation strategy used consists in obtaining the estimates for $\sigma^{2}$ and $\theta_{i i}, i=1,2$ and directly substitute them in (12). The plug-in rule $(P I)$ used was: $\sigma^{2}$ was estimated by $\hat{\sigma}^{2}=$ $\frac{1}{n} \sum_{i=1}^{n}\left(y_{t}-\widehat{m}_{1}^{b}\left(X_{i}\right)-\widehat{m}_{2}^{b}\left(Z_{i}\right)\right)^{2}$ where $\widehat{m}_{1}^{b}\left(X_{i}\right)$ and $\widehat{m}_{2}^{b}\left(Z_{i}\right)$ are the solutions to the backfitting algorithm given in (3) and $\widehat{\theta}_{11}$ and $\widehat{\theta}_{22}$ were estimated by equation (10) proposed by Opsomer \& Ruppert(1998), that is, $\hat{\theta}_{11}=\frac{1}{n} \operatorname{Tr} \mathbf{V}_{1}^{(2) *} \mathbf{Y} \mathbf{Y}^{\mathrm{T}} \mathbf{V}_{1}^{(2) * \prime}$ and $\hat{\theta}_{22}=$ $\frac{1}{n} \operatorname{Tr} \mathbf{V}_{\mathbf{2}}^{(2) *} \mathbf{Y Y}^{\prime} \mathbf{V}_{\mathbf{2}}^{(2) * \prime}$ where

$$
\begin{aligned}
V_{1}^{(2)} & =S_{1}^{(2)}\left(I_{n}-S_{2}^{*} S_{1}^{*}\right)^{-1}\left(I_{n}-S_{2}^{*}\right), V_{2}^{(2)}=S_{2}^{(2)}\left(I_{n}-S_{1}^{*} S_{2}^{*}\right)^{-1}\left(I_{n}-S_{1}^{*}\right) \\
V_{1}^{(2) *} & =\left(I-11^{\prime} / \mathbf{n}\right) V_{1}^{(2)}, V_{2}^{(2) *}=\left(I-11^{\prime} / n\right) V_{2}^{(2)}
\end{aligned}
$$

and $\mathbf{S}_{1}^{(2)}$ and $\mathbf{S}_{2}^{(2)}$ represent the matrices whose rows can be written as $\left(\mathrm{s}_{\mathbf{1}, \mathbf{x}}^{(\mathbf{2})}\right)^{\prime}=2 ! e_{3}^{4 \prime}\left(\mathbf{R}_{\mathbf{X}}(\mathbf{x})^{\prime} \mathbf{V}_{\mathbf{X}}(\mathbf{x}) \mathbf{R}_{\mathbf{X}}(\mathbf{x})\right)^{-1} \mathbf{R}_{\mathbf{X}}(\mathbf{x})^{\prime} \mathbf{V}_{\mathbf{X}}(\mathbf{x})$ and $\left(\mathrm{s}_{\mathbf{2}, \mathbf{z}}^{(\mathbf{2})}\right)^{\prime}=2 ! e_{3}^{\mathbf{4}}\left(\mathbf{R}_{\mathbf{Z}}(\mathbf{z})^{\prime} \mathbf{V}_{\mathbf{Z}}(\mathbf{z}) \mathbf{R}_{\mathbf{Z}}(\mathbf{z})\right)^{-1} \mathbf{R}_{\mathbf{Z}}(\mathbf{z})^{\prime} \mathbf{V}_{\mathbf{Z}}(\mathbf{z})$. The rule of thumb (ROT) described above was used to estimate the matrices $\mathbf{V}_{\mathbf{X}}(\mathbf{x})$ and $\mathbf{V}_{\mathbf{Z}}(\mathbf{z})$ which appear in $\left(\mathrm{s}_{1, \mathbf{x}}^{(2)}\right)^{\prime}$ and $\left(\mathrm{s}_{\mathbf{2}, \mathbf{z}}^{(\mathbf{2})}\right)^{\prime}$. 


\section{The Data Generating Process.}

The data used in the study were generated by a bivariate additive nonparametric regression model fitted by local linear regression, with varying correlation to evaluate the robustness to lack of independence between regressors. It is assumed that $\left\{\left(y_{t}, x_{t}, z_{t}\right)^{\prime}\right\}_{t=1}^{n}$ form a sequence of realizations of a $\Re^{3}$ - valued random vector $(\mathbf{Y}, \mathbf{X}, \mathbf{Z})$ and $\left\{\epsilon_{t}\right\}_{t=1}^{n}$ is a sequence of realizations of a random variable with distribution $N(0,1)$. The model used here can be described by

$$
Y_{t}=m_{1}\left(X_{t}\right)+m_{2}\left(Z_{t}\right)+\epsilon_{t}
$$

where $m_{1}\left(X_{t}\right)=-6 X_{t}+36 X_{t}^{2}-53 X_{t}^{3}+22 X_{t}^{5}, m_{2}\left(Z_{t}\right)=\operatorname{sen}\left(Z_{t}\right)$, $X_{t}=S_{t}, Z_{t}=5 \pi W_{t}$, with $\left\{W_{t}, S_{t}\right\}_{t=1}^{n}$ generated by a joint density function with the desired correlation, with marginals $N(1 / 2,1 / 9)$. Three levels of correlation were used to investigate robustness: 0 (independence), .25 ("low" correlation), .75 ("high" correlation).

The existence of a solution to the backfitting algorithm is generally unknown, but in the case in which local linear estimators are used, Opsomer \& Ruppert $(1997,1998)$ derived a series of sufficient conditions that guarantee the existence of a single solution in the bivariate case (see conditions A1 to A4 described in section 2).

Because of A2 we rejected all observations for which one of the regressors exceeded $\pm 1.5 \sigma$ of the mean (or equivalently outside the interval $[0,1])$, and in this case, we replaced them by new observations that fell within these limits. We considered samples of 100,150 and 200 observations, each of which was replicated 800, 600 and 400 times, respectively.

In this study, a Gaussian kernel was used. Some important results within this context are given next. For the Gaussian kernel, we 
obtain: $\mu_{1}\left(K_{(1)}\right)=0, \mu_{2}\left(K_{(1)}\right)=1$ and $R\left(K_{(1)}\right)=(2 \sqrt{\pi})^{-1}$.

\section{Results.}

A simulation study was carried out to evaluate and compare the performance of the B and MI-estimators in finite samples for a bivariate additive regression. Such study is necessarily restrictive because there are many possibilities regarding the selection of the regression function, the density of regressors, the correlation between them, the error density, the sample size, the type of polynominal regression, the kernel function, the chosen bandwidth, the type of squared error criterion function, among other factors.

By looking at figure 2 by Opsomer \& Ruppert(1997, p.191) we can note that the correlation 0.75 is outside the bounds set by assumption A2 of the referred article (p.190), when one normal bivariate distribution is used. Apparently, this does not affect the convergence. This supports the idea that correlation within these bounds, although sufficient, is not a necessary condition for the convergence of backfitting estimators. The kernel function used also does not satisfy condition A1. Likewise, this does not seem to affect the application of the results derived by Opsomer \& Ruppert(1997).

The primary aim of the article is to compare the performance of B and MI-estimators in finite samples. For this purpose, we computed the average squared error $A S E=\frac{1}{n} \sum_{t=1}^{n}\left(\widehat{m}_{1}\left(X_{t}\right)+\widehat{m}_{2}\left(Z_{t}\right)\right.$ $\left.-m_{1}\left(X_{t}\right)-m_{2}\left(Z_{t}\right)\right)^{2}$ in the simulation studies. After that, we calculated the mean of replications in order to estimate the $M A S E$. By comparing the values presented in Tables 1 and 2 we observed that B-estimators had a better performance than MI-estimators. 
Table 1. MASE estimates using backfitting with bandwidths $P I$ and the true $A M A S E$

\begin{tabular}{lllllll}
\hline \hline & \multicolumn{3}{c}{$h_{P I}$} & \multicolumn{3}{c}{$h_{\text {AMASE }}$} \\
& $n=100^{7}$ & $n=150$ & $n=200$ & $n=100$ & $n=150$ & $n=200$ \\
\hline$\rho=0$ & 0.606 & 0.470 & 0.397 & 0.327 & 0.284 & 0.261 \\
$\rho=0.25$ & 0.600 & 0.479 & 0.395 & 0.323 & 0.288 & 0.261 \\
$\rho=0.75$ & 0.596 & 0.470 & 0.399 & 0.321 & 0.279 & 0.260 \\
\hline
\end{tabular}

Table 2. $M A S E$ estimates using Marginal Integration with $R O T$ bandwidths and the true $A M A S E$

\begin{tabular}{lcccccc}
\hline & & $h_{\text {ROT }}$ & & \multicolumn{3}{c}{$h_{\text {AMASE }}$} \\
& $n=100$ & $n=150$ & $n=200$ & $n=100$ & $n=150$ & $n=200$ \\
\hline$\rho=0$ & 1.799 & 0.802 & 0.645 & 0.587 & 0.500 & 0.367 \\
$\rho=0.25$ & 5.133 & 1.036 & 3.173 & 0.694 & 0.460 & 0.385 \\
$\rho=0.75$ & 14.967 & 5.297 & 12.028 & 3.385 & 2.228 & 2.525 \\
\hline
\end{tabular}

By analyzing Tables 1 and 2, it is possible to observe a series of important facts. Firstly, note that the denial of the independence hypothesis between regressors does not affect the estimation made with the backfitting algorithm, no matter if the correlation between regressors is low $(\rho=0.25)$ or high $(\rho=0.75)$. This does not occur when the Marginal Integration is used. In this case, the impact of ignoring dependency remarkably influences the results obtained.

Also, note that the bandwidths used in this Monte Carlo investigation are chosen so as to minimize $M A S E$. Thus, the comparison between estimators should be made using the $M A S E$ criterion. However, if the median of the replications is used to compare the

${ }^{7}$ The bandwidth $h_{2 n P I}$ had overflow problems in the simulation study. The data-generating process was repeated once when $\rho=0$ and $\rho=0.75$ and twice when $\rho=0.25$. 
estimators the results show visible differences. The results obtained were somehow expected. Opsomer \& Ruppert(1997, p.198) comment that there is an interesting difference between both estimators when $\mathbf{X}$ and $\mathbf{Z}$ are independent. In this case, it is natural to expect that the asymptotic bias of estimators of an additive model for estimating one of the component functions does not depend on the behavior of the other function. Opsomer \& Ruppert(1997) show that the B estimator has such property, whereas the MI estimator does not. Except if the bias effects of the component functions happen to offset each other, this will likely result in an increased bias relative to the backfitting estimator. The comparison between asymptotic variances is more straightforward due to the similar format of the expressions for both estimators. In this case, it is possible to show that the asymptotic variance of B-estimators is always smaller than that of MI-estimators, unless $\mathbf{X}$ and $\mathbf{Z}$ are independent.

The comparison between both estimators is clearer when the true bandwidths $h_{1 n A M A S E}$ and $h_{2 n A M A S E}$ are used. In a simulation study like this nothing is unknown in (12), that is, there will be no "noise" inherent to the estimation process when the two estimators are compared. In this case, there noticeably exist strong signs of the superiority of B-estimators.

In an attempt to clarify the superiority of B-estimators, the $M A S E$ of these estimators was calculated using the bandwidths $h_{\text {inROT }}, i=1,2$, directly. These bandwidths were constructed in a format that is appropriate for the estimation via Marginal Integration. We suspect that even when using an appropriate rule for the estimation of MI-estimators, the performance of B-estimators would still be superior, which could be confirmed here. Nevertheless, something amazing occurred, as can be observed when we compare Tables 3 and 1 . Apparently, the estimation of the second derivate 
made in Opsomer \& Ruppert(1998) deteriorates the performance of B-estimators in finite samples instead of improving it. Albeit unexpected, the result is interesting, since little is known about the properties of this estimator in finite samples.

Table 3. $M A S E$ estimates using backfitting with $R O T$ bandwidth

\begin{tabular}{lccc}
\hline \hline & & $h_{\text {ROT }}$ & \\
& $n=100$ & $n=150$ & $n=200$ \\
\hline$\rho=0$ & 0.440 & 0.376 & 0.334 \\
$\rho=0.25$ & 0.438 & 0.365 & 0.329 \\
$\rho=0.75$ & 0.438 & 0.364 & 0.329 \\
\hline
\end{tabular}

Figures 1 and 2 show the densities ${ }^{8}$ of $\log \left(h_{\text {inAMASE }}\right)-$ $\log \left(h_{\text {inPI }}\right)$ and $\log \left(h_{\text {inAMASE }}\right)-\log \left(h_{\text {inROT }}\right), i=1,2$ for the levels of correlation used and for the samples sized 100, 150 and 200, each of which was replicated 800,600 and 400 times, respectively. As can be observed, the densities for the different levels of correlation are quite close. Seemingly the level of correlation between the covariates has little effect on the estimated bandwidths, which justifies the use of independence assumption in the computation of $h_{n P I}$ and $h_{n R O T}$. Estimator $h_{1 n P I}$ displays a small bias (undersmoothing) in the estimation of $m_{1}$ (low-degree polynomial) whereas estimator $h_{1 n R O T}$ has a stronger bias, causing an oversmoothing in the estimation of $m_{1}$. In this case, the estimators have a similar variability. Both estimators have a marked bias in the estimation of $m_{2}$ (undersmoothing), ${ }^{9}$ however the bias of estimators $h_{2 n P I}$ is stronger and also those densities display more variability. Estimators $h_{n R O T}$ have a similar variability in the estimation of $m_{1}$ and $m_{2}{ }^{10}$.

\footnotetext{
${ }^{8}$ Estimated by means of Sheather-Jones (1991) bandwidth.

${ }^{9}$ This is probably due to the fact that $m_{2}$ is a sine function (therefore less subject to first-order approximations than $m_{1}$ ).

${ }^{10}$ Note that the performance of both estimators improves as the sample size increases.
} 
Fernando A. Boeira Sabino da Silva
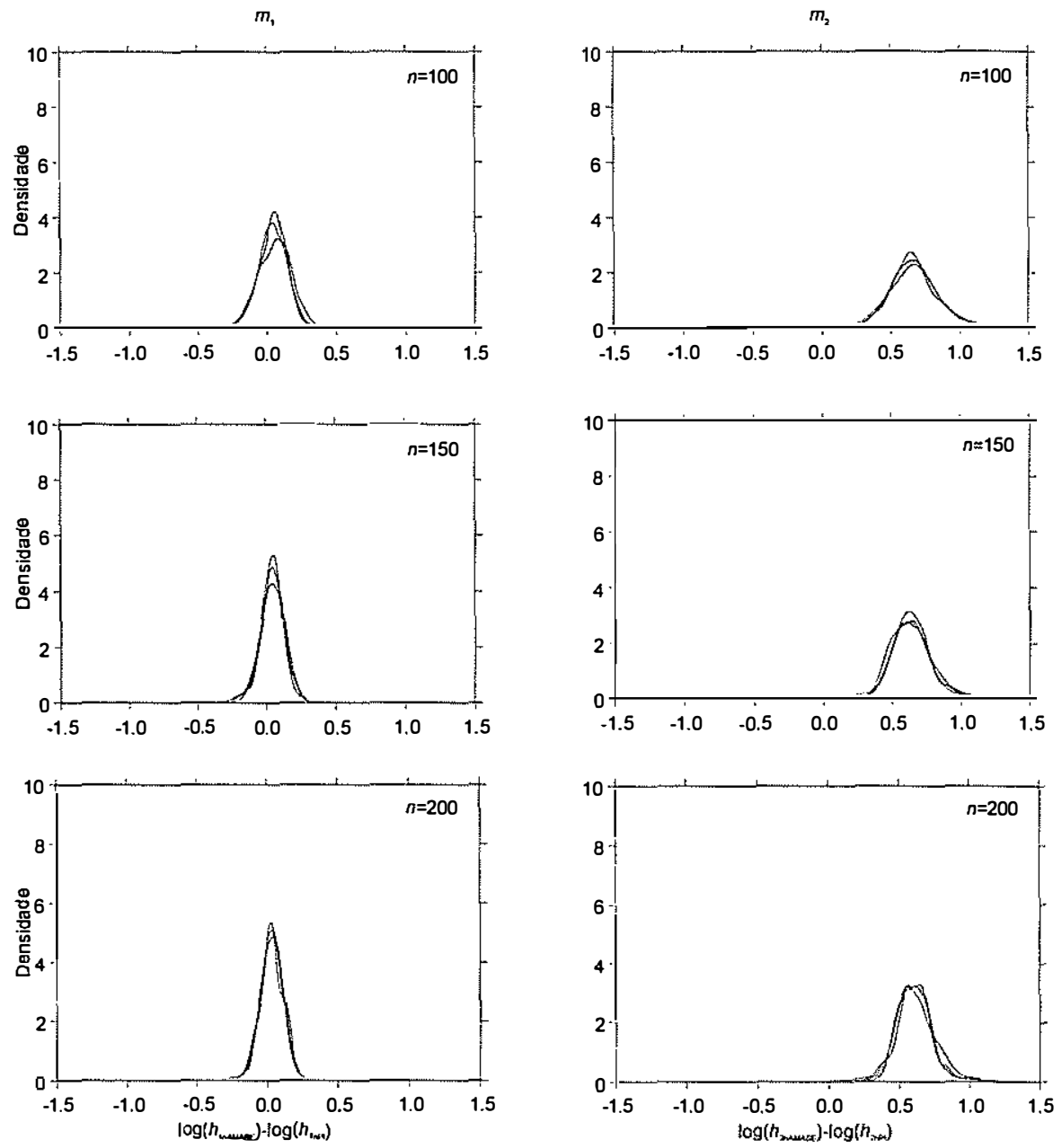

Figure 1: Density of the PI bandwidth estimators for the three levels of correlation 
Additive nonparametric regression estimation

$m$
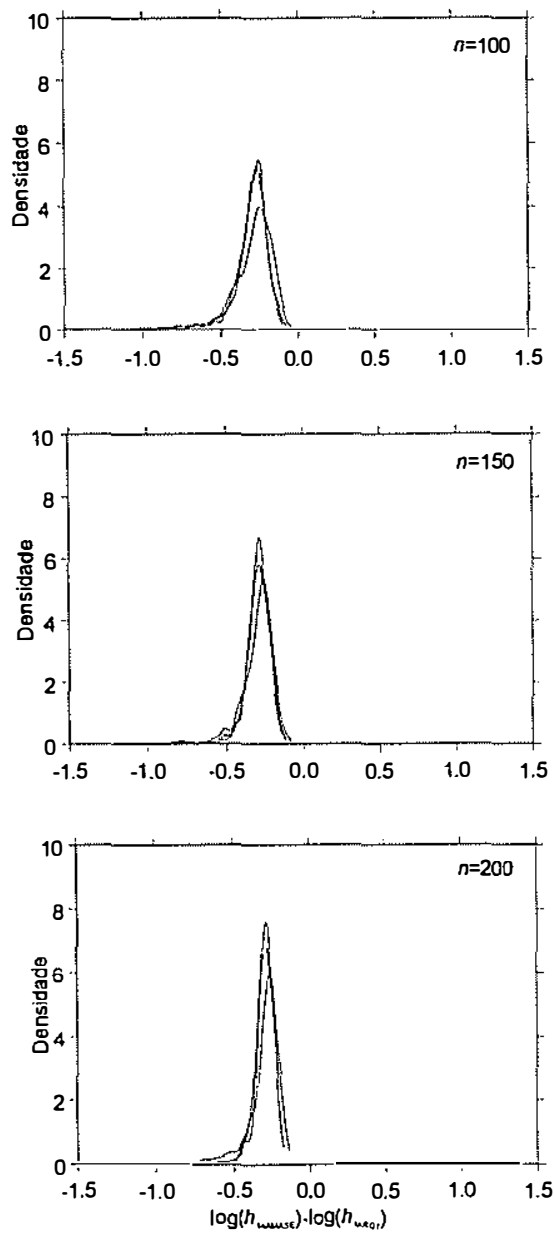

$m$,
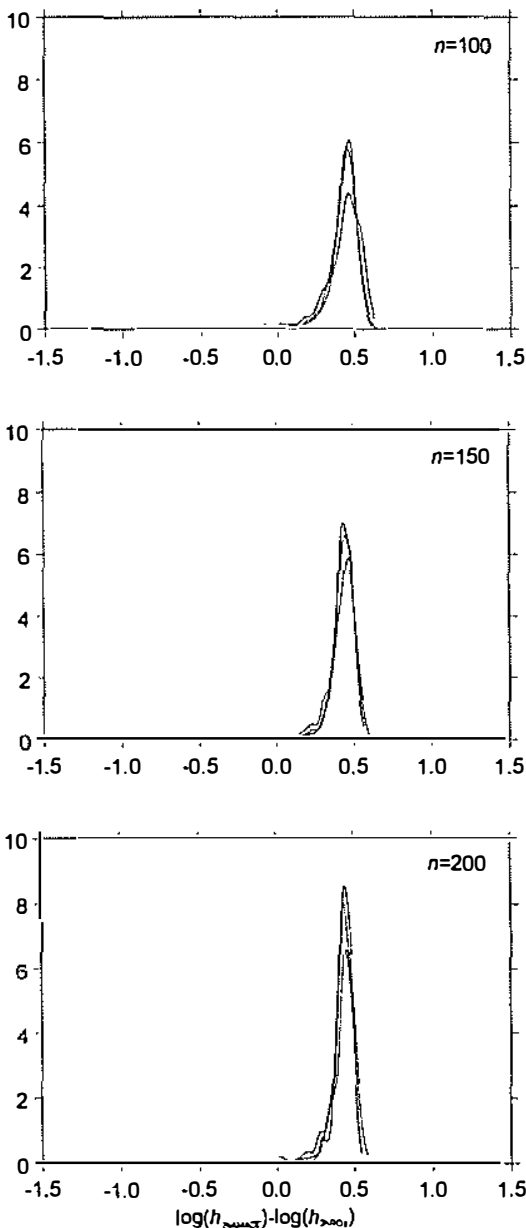

Figure 2: Density of the ROT bandwidth estimators for the three levels of correlation 


\section{Conclusions.}

The current literature proposes, basically, two methods for the estimation of an additive nonparametric regression: B and MI estimators. The comparison made by means of a Monte Carlo investigation suggests that the B-estimator has a superior performance to the MI- estimator. Although the simulation study presented here has a reduced scope, this is confirmed in a more comprehensive study, see Martins-Filho $(2001)^{11}$.

The estimator proposed by Linton \& Nielsen is based on an excellent idea, but it involves the product of the bandwidths. In the bivariate case, if the estimates for the two bandwidths are undersmoothed or oversmoothed the effect will be magnified. In addition, as mentioned in Silva(2001, p.16), the estimation via Marginal Integration is computationally more demanding ${ }^{12}$, which is inconvenient to the users. In fact, the MI-estimator presents problems associated with unrestricted multivariate regressions, which is undesirable.

The main objective of the article was to compare the two alternative estimation procedures for the estimation of an additive nonparametric regression. The main findings are summarized below.

1. The lack of the independence assumption between the regressors does not affect the estimation made via the backfitting al-

\footnotetext{
${ }^{11}$ Aside from the bandwidths used in this article, it is also used cross validation and the bandwidth $h_{D P I}$ (Opsomer \& Ruppert 1998). The latter is based on a plug-in method that apparently presents a better performance than the estimators used here.

${ }^{12}$ The difference between the simulation studies was remarkable. For samples sized 100 a replication with Marginal Integration lasted on average 33.7s. By using Backfitting with the bandwidth $P I$ it lasted on average 2.1 s and $1.2 \mathrm{~s}$ when the bandwidth $R O T$ was used. A Pentium III 500Mhz was used. The programs were created in Gauss version 3.6 and are available from the author upon request.
} 
gorithm. This does not happen when the Marginal Integration is used.

2. An interesting difference between the $B$ estimator and the MI estimator occurs when the $\mathbf{X}$ and $\mathbf{Z}$ regressors are independent. In this case, it is expected that the asymptotic bias of estimators of an additive model for estimating one of the component functions does not depend on the behavior of the other component. The Bestimator has such property, while the MII-estimator does not. For this reason, in general, the MI-estimator will present a stronger bias in relation to the B-estimator.

3. The asymptotic variance of B-estimators is always smaller than that of the MII-estimators, unless the regressors are independent.

4. In general, the MI-estimator needs to compute a higher number of operations than the B-estimator in order to estimate the additive components (see Kim, Linton and Hengartner, 1999), that is, the computational demand of the MI-estimator is greater than that of the B-estimator.

5. In the bivariate case, the MI-estimator involves the products of two bandwidths. If the estimates of the bandwidths are undersmoothed or oversmoothed the effect will be magnified. similarly to the curse of dimensionality.

Submitted in March 2002. Revised in September 2002.

\section{References}

Buja, A., Hastie, T. \& R. Tibshirani 1989. "Linear Smoothers and Additive Models(with discussion)". Ammals of Statistics, 17, 453-555. 
Friedman, J.H. \& W. Stuetzle1981. "Projection Pursuit Regression", Journal of the American Statistical Association, 76, 817823.

Härdle, W., P. Hall \& J. S. Marron 1988. "How Far Are Automatically Chosen Regression Smoothing Parameters From Their Optimum?", Journal of the American Statistical Association, 83, 86-95.

Hastie, T. J. \& R. J. Tibshirani 1986. "Generalized Additive Models". Statistical Science, 1, Nº3, 297-318.

Hastie, T. J. \& R. J.Tibshirani 1990. Generalized Additive Models. Chapman and Hall, Washington, DC.

Jones, M. C., J. S. Marron \& S.J. Sheater 1996. "A Brief Survey of Bandwidth Selection for Density Estimation". Journal of the American Statistical Association, 91, 401-407.

Kim, W., O. B. Linton,\& N. W. Hengartner 1999. "A Computationally Efficient Oracle Estimator for Additive Nonparametric Regression with Bootstrap Confidence Intervals". Journal of Computational and Graphical Statistics, 8 , 2, 279-297.

Linton, O. \& J.P. Nielsen 1995. "A Kernel Method of Estimating Structured Nonparametric Regression based on Marginal Integration". Biometrika, 82, 1, 93-100.

Linton, O. \& Härdle, W. 1996. "Estimation of additive regression models with known links". Biometrika, 83, 3, 529-540.

Martins-Filho, C.B. 2001. "Additive Nonparametric Regression Estimator via Backfitting and Marginal Integration: Finite Sample Performance". Working paper. OSU, Department of Economics.

Martins-Filho, C.B. \& O. Bin 1999. "Estimation of hedonic price functions via additive nonparametric regression". Working paper. OSU, Department of Economics. 
Opsomer, J.D. \& Ruppert, D. 1997. "Fitting a Bivariate Additive Model by Local Polynomial Regression", The Annals of Statistics, 25, 1, 186-211.

Opsomer, J.D. \& D. Ruppert. 1998. "A Fully Automated Bandwidth Selection Method for Fitting Additive Models", Journal of the American Statistical Association, 93, 442, 605-619.

Ruppert, D., S. J. Sheather, \& M. P. Wand, 1995. "An Effective Bandwidth Selector for Local Least Squares Regression", Journal of the American Statistical Association, 25, 186-211.

Ruppert, D. \& M. P. Wand 1994. "A Multivariate Locally Weighted Least Squares Regression", Annals of Statistics, 22, 1346-1370.

Sheater, S. J \& M. C. Jones 1991. "A reliable data-based bandwidth selection method for kernel density estimation". Journal of the Royal. Statistical Society, Series B, 53, 3, 683-690.

Silva, F. A. B. S. S. 2001. "Estimação de Regressões Aditivas via Backfitting e Integração Marginal: Performance em Amostras Finitas", Dissertação de Mestrado, Fundação Getúlio Vargas/EPGE, Rio de Janeiro, Brasil.

Silverman, B.W. 1986. Density Estimation for Statistics and Data Analysis. London: Chapman and Hall. 